\title{
Determining the Range of Noninvasive Near-Infrared Femtosecond Laser Pulses for Mammalian Oocyte Nanosurgery
}

\author{
DOI: 10.17691/stm2017.9.1.02
}

Received August 9, 2016

A.A. Osychenko, PhD Student ${ }^{1}$;

U.A. Tochilo, Student, Faculty of Biology2;

A.A. Astafiev, PhD, Senior Researcher ${ }^{1}$;

A.D. Zalessky, PhD, Senior Researcher ${ }^{1}$; Associate Professor ${ }^{3}$;

A.M. Shakhov, PhD, Junior Researcher ${ }^{1}$;

A.S. Krivokharchenko, PhD, Researcher ${ }^{1}$;

V.A. Nadtochenko, DSc, Professor, Deputy Director on Scientific Work ${ }^{1}$

${ }^{1}$ Semenov Institute of Chemical Physics of the Russian Academy of Sciences, 4 Kosygina St., Moscow, 119991, Russian Federation;

${ }^{2}$ Lomonosov Moscow State University, 1/12 Leninskye Gory, Moscow, 119991, Russian Federation;

${ }^{3}$ Moscow Institute of Physics and Technology (State University), 9 Institutskiy per., Dolgoprudny, Moscow region, 141701, Russian Federation

The aim of the investigation was to determine a noninvasive range of near-infrared femtosecond laser pulse exposure for mouse oocyte intracellular nanosurgery and to estimate oocyte developmental competence in vitro depending on pulse energy and total exposure time.

Materials and Methods. The model object was a preovulatory germinal vesicle mouse oocyte. Femtosecond laser radiation with a central wavelength of $780 \mathrm{~nm}$ was utilized in two modes: 1) single pulses with 30 fs duration and pulse energy up to $100 \mathrm{~nJ}$; 2) trains of pulses with 100 fs duration, $80 \mathrm{MHz}$ repetition rate (12.5 ns between pulses) and pulse energy from 0.5 to $2 \mathrm{~nJ}$; total duration of the pulse trains ranged from 15 to $60 \mathrm{~ms}$. Radiation was focused by a microscope objective lens (60x, $0.7 \mathrm{NA})$. Oocytes were cultured to the metaphase of the second meiotic division, which was used as invasiveness criteria. This stage was detected by polar bodies and metaphase plate formation.

Results. The threshold of invasiveness was determined for a single femtosecond pulse exposure. Cytoplasmic membrane rupture and cell disruption occurred under the exposure of a single $30 \mathrm{fs}$ pulse with $100 \mathrm{~nJ}$ energy. The cytoplasm and nucleus exposure to femtosecond pulse trains did not induce permanent damage to the oocytes. The rate of gas-vapor bubble formation was measured after the action of pulse trains on the cytoplasm and nucleus chromatin of mammalian oocytes. The pulse energy of $1 \mathrm{~nJ}$ is a threshold value for bubble formation in the cytoplasm, and $2 \mathrm{~nJ}$ in the nucleus. Exposure to laser pulse trains with $80 \mathrm{MHz}$ repetition rate and $100 \mathrm{fs}$ duration didn't change oocyte ability to achieve metaphase II stage within the studied range of pulse energies and pulse train durations.

Conclusion. Surgery with femtosecond pulse trains at $80 \mathrm{MHz}$ repetition rate and pulse energy not more than twice as high as the threshold of cavitation or vapor bubble formation does not affect oocyte development to the metaphase II stage. Femtosecond laser nanosurgery can be regarded as a minimally invasive method of intracellular surgery within the investigated range of pulse energies and exposure times.

Key words: intracellular surgery; laser surgery; femtosecond laser; preovulatory oocytes.

Nanosurgical operations on the cells and embryos using femtosecond lasers with the wavelength in the near-infrared range is a challenging direction of modern biophotonics. The near-infrared range is in the region of biotissue transparency: it is not absorbed either by the water or organic substances of the cell (proteins, lipids, nucleic acids, carbohydrates) [1]. A high localization of the femtosecond pulse action in the area of laser beam waist (about 10 femtoliters volume) allows one to perform operations inside the cell/embryo on a separate organelle leaving intact the cell environment and causing no damage to the outer membrane [1-3]. Non-linear optical absorption of laser pulse energy by the cell material is achieved at relatively low energies of femtosecond pulse, as a high light power density necessary for non-linear optical interactions is provided by a short pulse duration and tight focusing by the microscope objective lens. Low-energy pulses prevent significant thermal stress. Thus, a focused femtosecond laser can be used as a unique scalpel, which allows the

For contacts: Alina A. Osychenko, e-mail: alina.chemphys@gmail.com 
operations to be performed with submicron accuracy inside the cell/embryo without any damage to the object outer membranes [1-4].

Absorption of femtosecond laser pulse energy is accompanied by ionization and dissociation of the cell material molecules, which can cause generation of the products toxic for the cell [4]. Absorption of femtosecond laser pulse leads also to generation of acoustic or shock waves propagating from the beam waist region and causing cavitation - generation of hollow bubbles in the liquid medium. At a high laser radiation intensity and $80 \mathrm{MHz}$ repetition rate a strong heating of the liquid in the focal point can occur, resulting in boiling and generation of gas-vapor bubbles. If the laser pulse energy exceeds a certain level, these processes may damage the cell structure. The effect of these unfavorable factors on the cell/embryo viability and developmental competence is poorly studied so far.

The aim of the investigation was to determine the range of noninvasive effect of near-infrared femtosecond laser pulses tightly focused in the cytoplasm or chromatin of the mammalian germinal vesicle oocyte, and to assess the developmental competence of oocytes in vitro depending on the pulse energy and total dose of the incident radiation.

Materials and Methods. Preovulatory mouse oocytes isolated at the germinal vesicle stage were chosen as a model system.

Oocyte preparation. Hybrid female mice (F1 line, CBA/C57B1) aged from 1.5 to 2.5 months were used to produce oocytes. The protocol of oocyte isolation is described in work [3].

The work was performed in accordance with ethical principles established by European Convention for the Protection of Vertebrata used for Experimental and other Scientific Purposes (the Convention was passed in Strasburg, March 18, 1986, adopted in Strasburg, June 15, 2006) and approved by Ethics Committee of Semenov Institute of Chemical Physics of the Russian Academy of Sciences.
Oocytes cleaned from cumulus cells were placed in a $50 \mu \mathrm{l}$ drop of Sigma-M7167, M2 medium (SigmaAldrich, USA) on the cover glass. A control group (without laser exposure) of oocytes was prepared in the same way. The time spent for manipulations with the sample during laser irradiation did not exceed $1 \mathrm{~min}$. After the manipulation, the oocytes were washed in $\mathrm{M} 2$ medium, cultivated in $\mathrm{CO}_{2}$-incubator $\left(5 \% \mathrm{CO}_{2}, 37^{\circ} \mathrm{C}\right)$ in the medium for in vitro maturation. The medium was prepared on the basis of DMEM C420 medium (PanEco, Russia) adding $15 \%$ fetal bovine serum (I31966-021; Gibco, USA), Gentamycin antibiotic (G1272; SigmaAldrich, USA) at $1.5 \mathrm{ME} / \mathrm{ml}$ concentration and PMSG1 $\mathrm{ME} / \mathrm{ml}$ hormone (A036A02; Intervet, Germany) [5]. Observation of the development was started the next day $18 \mathrm{~h}$ after the operation.

Schematic diagram of the device. Femtosecond laser radiation with a wavelength of $780 \mathrm{~nm}$ was utilized in two modes: 1) single pulses with 30 fs duration and pulse energy up to $100 \mathrm{~nJ} ; 2$ ) trains of pulses with $100 \mathrm{fs}$ duration, $80 \mathrm{MHz}$ repetition rate $(12.5 \mathrm{~ns}$ between pulses) and pulse energy from 0.5 to $2 \mathrm{~nJ}$; total duration of the pulse trains was between 15 and $60 \mathrm{~ms}$. The principle scheme of femtosecond laser scalpel is presented on Figure 1. The detailed scheme of the device has been described previously [3].

Laser radiation was focused by $60 \times, 0.7$ NA (numerical aperture) objective lens. The spot had the following parameters: laser beam waist $w_{0}=0.61 \mathrm{~N} / \mathrm{NA}$, $\mathrm{w}_{0}=0.68 \mu \mathrm{m}$; Rayleigh parameter $\mathrm{z}_{0}=\mathrm{kw}_{0}{ }^{2} / 2, \mathrm{z}_{0}=1.86 \mu \mathrm{m}$ $\left(k=2 p / l_{0}\right.$ - wave number). Laser pulse energy was varied using polarization attenuator. Total dose of $80 \mathrm{MHz}$ laser pulses was changed by altering pulse train duration using a laser beam mechanical shutter. Femtosecond laser radiation was focused either at a randomly selected point of the cytoplasm or in the chromatin area in the germinal vesicle (nucleus). There were 10 oocytes for every pair of exposure parameters (duration of pulse train and the energy of each pulse). Laser exposure was repeated 5 times, so there were 50 pulse trains for 10 samples,

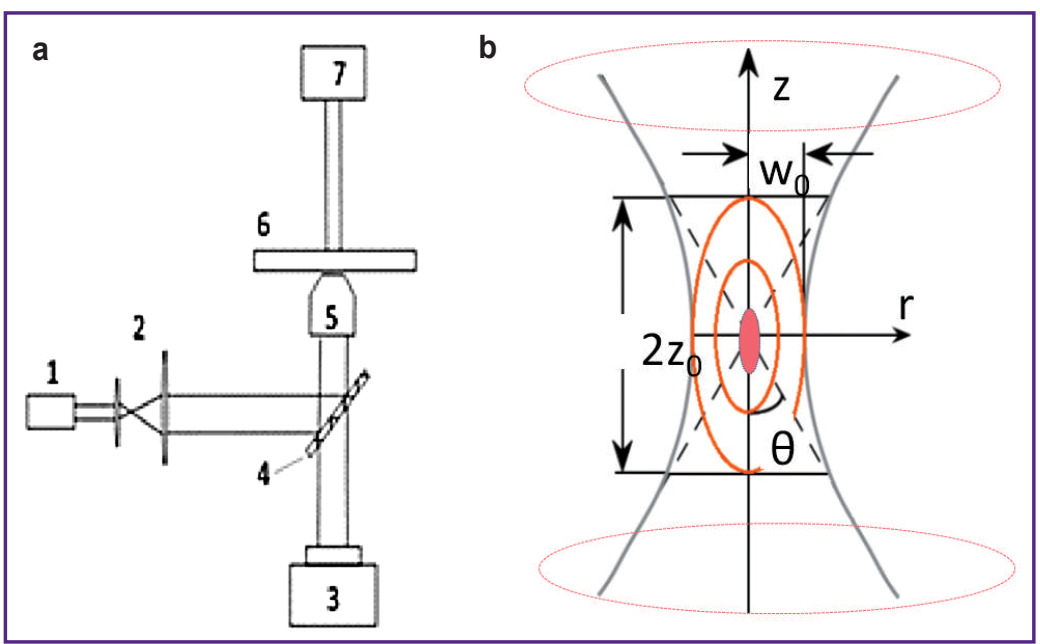

Figure 1. Principle schematic diagram of femtosecond laser scalpel: (a) optical scheme: femtosecond Ti:Sapphire laser (1), telescope - laser beam expander (2), light source (3), dichroic mirror (4), microscope objective (5), object stage (6), video camera (7); (b) laser beam waist: Rayleigh parameter $\mathrm{z}_{0}$ and waist radius $\mathrm{w}_{0}$; the envelope shown by a solid line corresponds to level $1 / \mathrm{e} \approx 1 / 2.71828$ of the bright field intensity $E$ 
and the total exposure amounted to 5 trains. During the repeated impact the focal spot was randomly moved inside the oocyte plasma or nucleus. The alteration of the object morphology after laser exposure was registered in the form of a video file using DCC1545M camera (Thorlabs, USA).

Fluorescence localization. Isolation of polar bodies, testifying to the accomplishment of oocyte maturation, and formation of metaphase plates were registered using bright field technique and fluorescence microscopy. Oocytes were stained with Hoechst 33342 stain (B2261; Sigma-Aldrich, USA), which binds to DNA. Images were acquired by means of Biomed 4 PR LUM microscope (Biomed, Russia) and Ximea xiD MD061CU-SY camera (Ximea, Slovakia).

Statistical data processing. To assess the significance of the difference between experimental and control groups, exact Fisher criterion has been used, as it is the most appropriate test for small sample size and the probability can be calculated exactly rather than relying on approximation.

Results. When exposing the nucleus (Figure 2) or cytoplasm (Figure 3) to laser pulses, gasvapor and cavitation bubbles may be generated. Bubbles are generated in a stochastic manner. The bubble generation rate as a function of laser pulse energy and pulse train duration at $80 \mathrm{MHz}$ repetition rate are presented in Table 1. Different areas of oocyte (nucleus and cytoplasm) have various threshold of gas-vapor bubble formation. The number of gas-vapor bubbles generated under the action of these 50 pulse trains on the nucleus and cytoplasm was calculated. The probability of gas-vapor bubble generation in the cytoplasm turned out to be essentially higher at the same parameters.
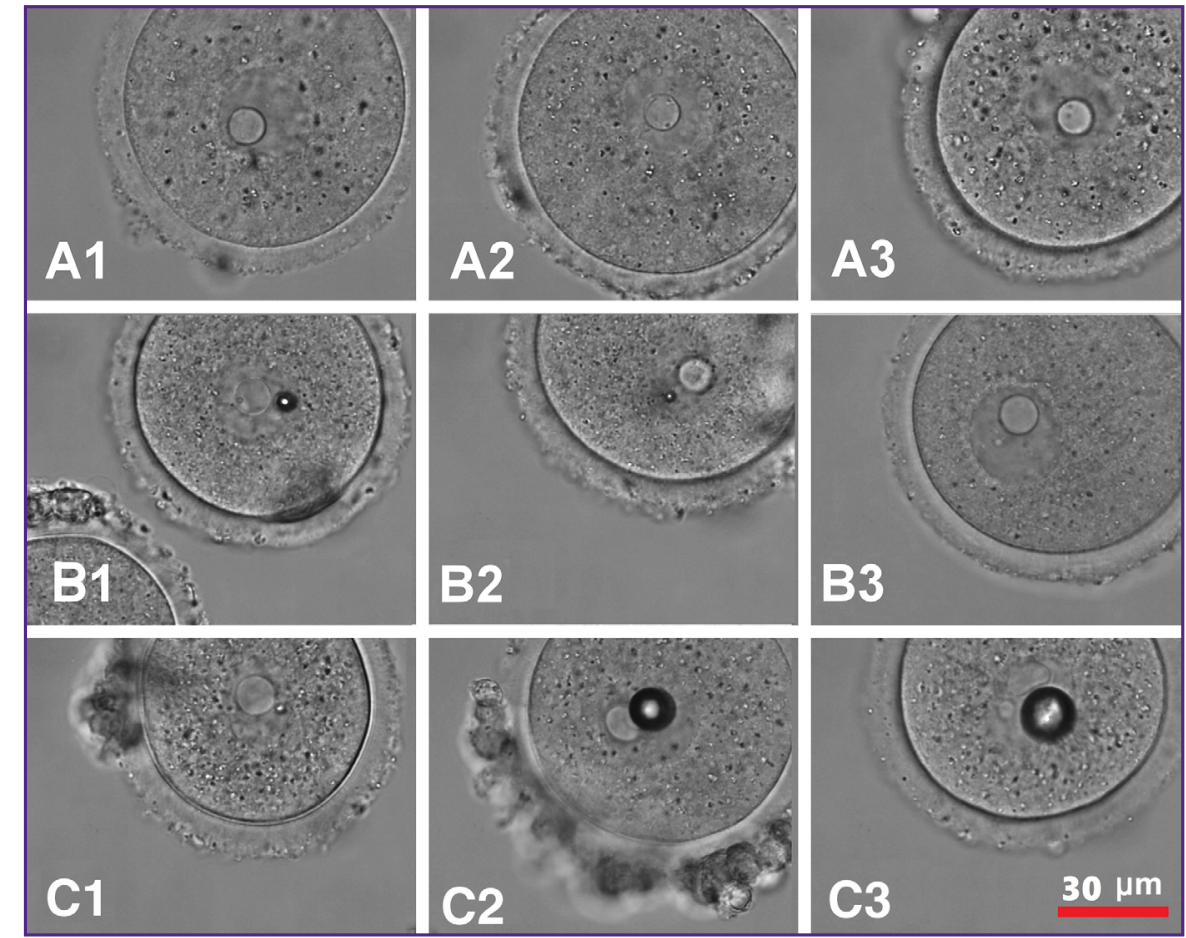

Figure 2. Shots from video records showing the generation of gas-vapor bubbles when focusing in the nucleus: (A1) pulse energy $0.5 \mathrm{~nJ}$, pulse train duration $15 \mathrm{~ms}$; (A2) $0.5 \mathrm{~nJ}, 30 \mathrm{~ms}$; (A3) $0.5 \mathrm{~nJ}, 60 \mathrm{~ms}$; (B1) $1 \mathrm{~nJ}, 15 \mathrm{~ms}$; (B2) $1 \mathrm{~nJ}, 30 \mathrm{~ms}$; (B3) $1 \mathrm{~nJ}$, $60 \mathrm{~ms}$; (C1) $2 \mathrm{~nJ}, 15 \mathrm{~ms} ;(\mathrm{C} 2) 2 \mathrm{~nJ}, 30 \mathrm{~ms}$; (C3) $2 \mathrm{~nJ}, 60 \mathrm{~ms}$
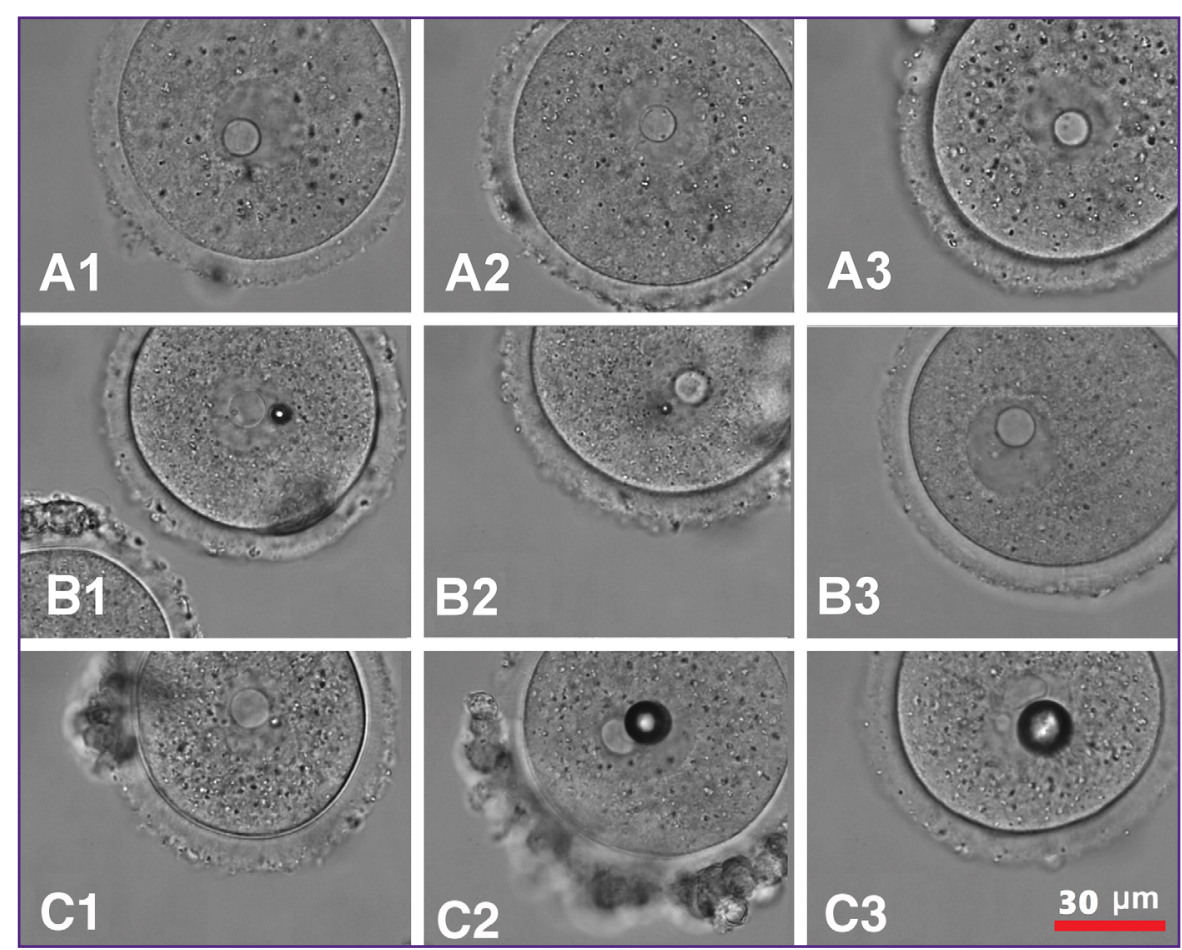

Figure 3. Shots from video records with generation of gas-vapor bubbles when focusing in the cytoplasm: (A1) pulse energy $0.5 \mathrm{~nJ}$, pulse train duration $15 \mathrm{~ms}$; (A2) $0.5 \mathrm{~nJ}, 30 \mathrm{~ms}$; (A3) $0.5 \mathrm{~nJ}, 60 \mathrm{~ms}$; (B1) $1 \mathrm{~nJ}, 15 \mathrm{~ms}$; (B2) $1 \mathrm{~nJ}, 30 \mathrm{~ms}$; (B3) $1 \mathrm{~nJ}$, 60 ms; (C1) 2 nJ, 15 ms; (C2) 2 nJ, 30 ms; (C3) 2 nJ, 60 ms 
Table 1

The rate of gas-vapor bubble generation in the nucleus and cytoplasm of preovulatory mouse oocytes at various exposure parameters

\begin{tabular}{ccc}
\hline $\begin{array}{c}\text { Pulse energy } \\
\left(\begin{array}{c}\text { (nJ)/pulse train } \\
\text { duration (ms) }\end{array}\right.\end{array}$ & $\begin{array}{c}\text { The number of bubble generation events } \\
\text { per quantity of laser exposure tests (50 trains) }\end{array}$ \\
\hline $0.5 / 15$ & 0 & 0 \\
Impact in the nucleus & Impact in the cytoplasm \\
\hline $0.5 / 30$ & 0 & 0 \\
\hline $0.5 / 60$ & 0 & 0 \\
\hline $1 / 15$ & 4 & 15 \\
\hline $1 / 30$ & 3 & 15 \\
\hline $1 / 60$ & 5 & 30 \\
\hline $2 / 15$ & 3 & 13 \\
\hline $2 / 30$ & 6 & 30 \\
\hline $2 / 60$ & 10 & 32 \\
\hline
\end{tabular}

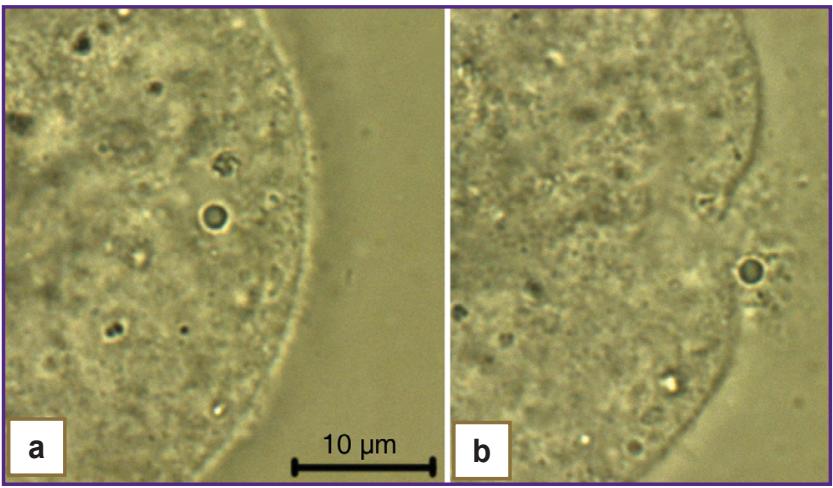

Figure 4. Shots from video record showing rupture of the oocyte cytoplasmic membrane due to generation of the cavitation bubble following a single pulse impact with the energy of $100 \mathrm{~nJ}$ on the cytoplasm: (a) prior to laser exposure; (b) $20 \mathrm{~s}$ after laser exposure

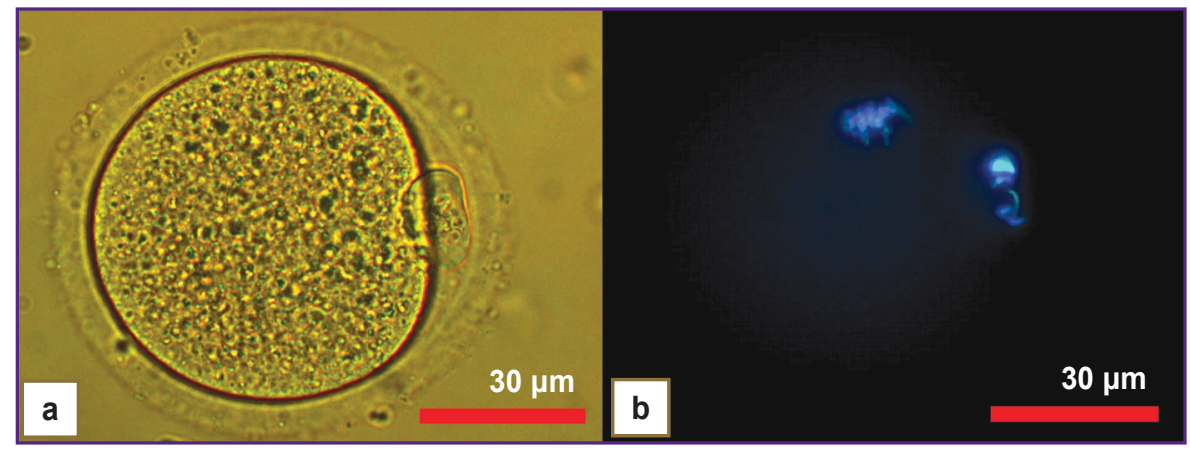

Figure 5. Oocyte at the stage of metaphase II: (a) image in the transmitted light; (b) fluorescent image (fluorescence of the metaphase plate from the top and the polar body on the right are seen)

Table 2

Development of preovulatory oocytes up to metaphase II stage after exposure of various cell areas (nucleus and cytoplasm) to a femtosecond laser

\begin{tabular}{ccc}
\hline $\begin{array}{c}\text { Pulse energy } \\
\text { (nJ)/pulse train } \\
\text { duration (ms) }\end{array}$ & $\begin{array}{c}\text { The number of oocytes reached the stage of metaphase II/number } \\
\text { of oocytes in the group (percentage of oocytes at the stage of metaphase II } \\
\text { of the total number); } p \text {-value (exact Fisher test) }\end{array}$ \\
\hline $0.5 / 15$ & $\begin{array}{c}\text { Impact in the nucleus } \\
\text { Impact in the cytoplasm }\end{array}$ \\
\hline $0.5 / 30$ & $8 / 20(40 \%) ; 0.99$ & $14 / 30(47 \%) ; 0.66$ \\
\hline $0.5 / 60$ & $9 / 20(45 \%) ; 0.80$ & $11 / 30(37 \%) ; 0.82$ \\
\hline $1 / 15$ & $10 / 28(36 \%) ; 0.82$ & $15 / 30(50 \%) ; 0.39$ \\
\hline $1 / 30$ & $7 / 30(23 \%) ; 0.11$ & $7 / 20(35 \%) ; 0.79$ \\
\hline $1 / 60$ & $7 / 30(23 \%) ; 0.11$ & $9 / 30(30 \%) ; 0.37$ \\
\hline $2 / 15$ & $7 / 21(33 \%) ; 0.62$ & $16 / 40(40 \%) ; 0.99$ \\
\hline $2 / 30$ & $14 / 30(47 \%) ; 0.66$ & $10 / 30(50 \%) ; 0.39$ \\
\hline $2 / 60$ & $10 / 30(33 \%) ; 0.65$ & $10 / 20(50 \%) ; 0.45$ \\
\hline Control & $14 / 30(47 \%) ; 0.66$ & \\
\hline
\end{tabular}

Exposure of cytoplasm or nucleus to a single pulse of more than $100 \mathrm{~nJ}$ results in a pressure rise in the laser waist center, the pressure wave reaches the cell plasma membrane and ruptures it (Figure 4). Membrane rupture induced by intensive laser pulse inside the oocyte causes cell death and will not be considered further.

After femtosecond laser exposure by $80 \mathrm{MHz}$ pulse trains, oocytes are able to develop in vitro up to metaphase II stage. Formation of the polar body was registered after $18 \mathrm{~h}$ of cultivation. The criterion of reaching metaphase II stage was separation of the polar body and generation of the metaphase plate, the presence of which was registered by fluorescence of Hoechst 33342 stain (Figure 5). The oocytes reached the stage of metaphase II (Table 2) were counted in each group. Exact Fisher test did not found statistically significant difference between the experimental and control groups in the ability to reach metaphase $I I(p=0.05)$.

Discussion. A gas-vapor bubble forms in the area of laser focal spot inside the oocyte, i.e. the area of maximal laser radiation intensity. Absence of the marked dependence of the probability of bubble generation 
on the pulse train duration at constant pulse energy indicates that under the action of the pulse sequence, accumulation of color centers capable of absorbing radiation at $780 \mathrm{~nm}$ wavelength is negligible in the experiments conducted [1-4]. Based on the data of Table 1, a threshold of bubble generation (the probability of the event approximates to $50 \%$ ) in the cytoplasm is lower than in the nucleus. Bubble generation threshold is close to $1 \mathrm{~nJ}$ pulse energy in the cytoplasm.

In the nucleus this threshold is close to $2 \mathrm{~nJ}$. Decrease of the threshold is supposed to be connected either with a lower potential of the cytoplasm material ionization, or a high concentration of various organelles in the cytoplasm.

Cytoplasm organelles may be considered as dielectric globules from 1 to $5 \mu \mathrm{m}$ in diameter placed in the water. Enhancement of electromagnetic field intensity of the laser pulse occurs in the near field of organelles. Using our method of calculating the intensity of field strength $[6,7]$, the coefficient of field intensity enhancement was obtained, which appeared to be close to 5 , and the size of the near-field zone less than $0.5 \mu \mathrm{m}$. Intensity enhancement in the organelle near field may lead to the cytoplasm rupture by a focused laser pulse with formation of a bubble. At the femtosecond pulse energies from 0.5 to $2 \mathrm{~nJ}$, the intensity of the laser light in the beam waist is in the range of $3.4 \cdot 10^{11}$ to $13.6 \cdot 10^{11} \mathrm{~W} / \mathrm{cm}^{2}$, respectively. A threshold of bubble generation in the cytoplasm is close to the intensity of $6 \cdot 10^{11} \mathrm{~W} / \mathrm{cm}^{2}$. A threshold of water breakdown by a femtosecond pulse lies in the range of 6.6 to $9.0 \cdot 10^{12} \mathrm{~W} / \mathrm{cm}^{2}$ [8], i.e. and order higher than it is observed in the present work.

$780 \mathrm{~nm}$ wavelength used in this work is in the region of biotissue transparency, the linear absorption of light by an oocyte in this range is negligible [1-4]. Absorption of femtosecond pulse energy resulting in cavitation bubble generation is caused by nonlinear optical effects. Photoionization of the oocyte substance and formation of a low-density plasma may occur inside the bubble formation region [4]. When oocyte organic molecules are ionized, radicals and electrons must be formed. These intermediate substances react with oxygen producing as the final product, molecules of reactive oxygen species (ROS) $[1,4]$.

Formation of ROS takes place in the ionization volume which corresponds to the volume of the laser beam waist and is estimated as $5.4 \cdot 10^{-12} \mathrm{~cm}^{3}$. The volume of oocyte with $70 \mu \mathrm{m}$ in diameter is about $1.8 \cdot 10^{-7} \mathrm{~cm}^{3}$, the concentration of ionized molecules in the waist area equals to $10^{20} \mathrm{~cm}^{-3}$. In order to calculate an average ROS concentration after a single pulse impact on oocyte, the waist volume should be multiplied by the concentration of ions in the waist and divided by the oocyte volume. Thus, the average ROS concentration per oocyte is equal to $1.6 \cdot 10^{-6} \mathrm{M}$. The absence of significant difference between the experimental and control groups in the ability to reach metaphase II $(p>0.05)$ after laser exposure indicates to the fact that generation of ROS after 5 -fold impact on oocyte does not reduce its ability to maturate up to metaphase II.

So, femtosecond laser radiation of near-infrared range $(780 \mathrm{~nm})$ in the biological tissue transparency region enables physicians to perform nanosurgical operations inside the oocyte without disrupting cytoplasmic membrane and zona pellucida. Cutting the cell material with a femtosecond laser with $80 \mathrm{MHz}$ repetition rate is accompanied by cavitation and gasvapor bubble generation. When focusing inside the cytoplasm, a threshold of bubble generation is close to $6 \cdot 10^{11} \mathrm{~W} / \mathrm{cm}^{2}$, while focusing in the nucleus chromatin it is about two times higher. The impact on the cell material by the pulse trains with duration up to $60 \mathrm{~ms}$ and energy lower the threshold of bubble generation is not connected with formation of color centers in the cell. Oocyte surgery under such conditions does not influence the development of the oocyte to the metaphase II stage.

Conclusion. The range of noninvasive effect of the near-infrared femtosecond laser pulses has been determined. Action of single femtosecond pulses with averted energy results in sample destruction. Exposure to the femtosecond pulse trains does not influence the integrity of oocytes and their competence to further development in vitro. Femtosecond laser nanosurgery may be regarded as a minimally invasive method of intracellular surgery within the studied pulse energy and total exposure time range.

Study Funding. The work was supported by the grant of the Ministry of Education and Science, agreement on subsidy No.14.604.21.0058 (unique project identifier RFMEFI60414X0058).

Conflicts of Interest. The authors declare no conflicts of interest related to this study.

\section{References}

1. Nadtochenko V.A., Mel'nikov M.Ya. Lazernaya nanokhirurgiya kletok i embrionov. $\mathrm{V}$ kn.: Sintez, stroenie $i$ svoystva metall/poluprovodnik soderzhashchikh nanostrukturirovannykh kompozitov [Laser nanosurgery of the cells and embryos. In: Synthesis, structure and properties of metal/semiconductor-containing nanostructured composites]. Pod red. Trakhtenberg L.I., Mel'nikova M.Ya. [Trakhtenberg L.I., Mel'nikov M.Ya. (editors)]. Moscow: Tekhnosfera; 2016; p. 441-473.

2. Nuzzo V., Maxwell I., Chung S., Mazur E., Heisterkamp A. Subcellular surgery and nanoneurosurgery using femtosecond laser pulses. In: Biophotonics: spectroscopy, imaging, sensing, and manipulation. DiBartolo B., Collins J. (editors). Springer; 2011; p. 203-218, https://doi.org/10.1007/978-90-481-9977-899.

3. Osychenko A.A., Zalesskii A.D., Krivokharchenko A.S., Zhakhbazyan A.K., Ryabova A.V., Nadtochenko V.A. Fusion of blastomeres in mouse embryos under the action of femtosecond laser radiation. Efficiency of blastocyst formation and embryo development. Quantum Electronics 2015; 45(5): 498-502, https://doi.org/10.1070/ qe2015v045n05abeh015767. 
4. Vogel A., Noack J., Hüttman G., Paltauf G. Mechanisms of femtosecond laser nanosurgery of cells and tissues. Applied Physics B 2005; 81(8): 1015-1047, https://doi.org/10.1007/ s00340-005-2036-6.

5. Shishova K.V., Khodarovich Y.M., Lavrentyeva E.A., Zatsepina O.V. High-resolution microscopy of active ribosomal genes and key members of the rRNA processing machinery inside nucleolus-like bodies of fully-grown mouse oocytes. Experimental Cell Research 2015; 337(2): 208-218, https:// doi.org/10.1016/j.yexcr.2015.07.024.

6. Shakhov A.M., Astafiev A.A., Plutenko D.O., Sarkisov O.M., Shushin A.I., Nadtochenko V.A. Femtosecond optical trap-assisted nanopatterning through microspheres by a single Ti:Sapphire oscillator. The Journal of Physical Chemistry C 2015; 119(22): 12562-12571, https://doi.org/10.1021/acs. jpcc.5b00478.

7. Astaf'ev A.A., Shakhov A.M., Sarkisov O.M., Nadtochenko V.A. Microstructuring of polymer films by femtosecond pulses through optically trapped polystyrene microspheres. Quantum Electronics 2013; 43(4): 361-364, https://doi.org/10.1070/qe2013v043n04abeh015114.

8. Sarpe C., Köhler J., Winkler T., Wollenhaupt M., Baumert T. Real-time observation of transient electron density in water irradiated with tailored femtosecond laser pulses. New Journal of Physics 2012; 14(7): 075021, https://doi. org/10.1088/1367-2630/14/7/075021. 